\title{
Formulation Development and In vitro Evaluation of Oral Extended- release Capsules Containing Biodegradable Microspheres
}

\section{Gerald G Enriquez ${ }^{1 \#}$, Brandon A Orawiec ${ }^{1 \#}$, Syed AA Rizvi² and Duc P Do ${ }^{1 *}$}

${ }^{1}$ Department of Pharmaceutical Sciences, College of Pharmacy, Chicago State University, 9501 South King Drive, Chicago, IL 60628, USA

${ }^{2}$ Department of Pharmaceutical Sciences, College of Pharmacy, Nova Southeastern University, 3200 South University Drive, Fort Lauderdale, FL 33328, USA

\#These authors contributed equally to the work

\begin{abstract}
Oral extended-release delivery of Biopharmaceutics Classification System (BCS) class II and III compounds is desirable in order to decrease dosing frequency and potential adverse effects. Maintaining an extended-release profile of the drug is ideal to minimize fluctuations in plasma concentrations. The main objective of this research was to formulate a polymeric microsphere drug delivery system that will effectively provide extended-release characteristics via an orally administered capsule. Using acetaminophen and ibuprofen as model drugs, this drug delivery system was shown to provide extended-release characteristics of the drug for enhanced efficacy. The biodegradable polymeric matrix was formulated using bovine serum albumin (BSA) with glutaraldehyde as the crosslinker. The drug of interest was then encapsulated into the polymeric matrix and spray-dried into microspheres. Once prepared, the drug-loaded microsphere powder was placed into hard gelatin capsules. The physicochemical properties of the microsphere formulations were characterized. Additionally, differential scanning calorimetry was utilized to examine the thermal stability of the encapsulated drug. Release studies were conducted in vitro to examine the release profile of the drug. Cell uptake studies were carried out to examine the internalization capability of microsphere formulations. Drugloaded microspheres were found to be approximately 2 microns in size and exhibited a uniform size distribution. Zeta potential measurements were shown to be approximately $-30 \mathrm{mV}$, which indicated that the microspheres are stable in dispersions. Release data showed a constant release of the drug from the microsphere and capsule formulations for at least 16 hours. Formulated microspheres were able to be internalized into human intestinal Caco- 2 cells. These data provided evidence for the role of the microsphere delivery system in the extended-release delivery of BCS Class II and III drug substances.
\end{abstract}

Keywords: Microspheres; Oral delivery; Capsules; Extended-release; Dissolution; Caco-2

\section{Introduction}

All drug substances require a delivery system for transport, maintenance of stability, and delivery into the body. Advancements in the field of pharmaceutics have led to the development of a variety of dosage forms and drug delivery systems [1-3]. Despite major technological advancements in the field, oral administration via tablets and capsules remains the most convenient and preferred route for drug delivery due to its non-invasive nature, low cost, and patient compliance [4]. Although oral administration is desired, effective drug delivery continues to be challenging. The nature of the gastrointestinal (GI) tract, including the high enzymatic activity and acidic environment of the stomach can hinder absorption [5]. Additionally, drugs that exhibit a short half-life must be administered in multiple daily doses, which can be inconvenient and have a negative effect on patient compliance. There is an inverse correlation between the frequency of dosing and patient acceptance and compliance [6,7]. Consequently, the development of oral extended-release delivery system technologies and dosage forms with enhanced efficacy and bioavailability is highly desired. Oral extendedrelease delivery of Biopharmaceutics Classification System (BCS) class II and III compounds is desirable in order to decrease dosing frequency and potential adverse effects. Maintaining an extended-release profile of the drug is ideal to minimize fluctuations in plasma concentrations [7]. These fluctuations can result in either toxic or subtherapeutic effects for the patients. In addition, oral extended-release drug preparations should enhance convenience and patient compliance.

Oral extended-release dosage forms have been developed and commercially available for a number of drugs. Technologies currently utilized to produce extended-release delivery systems include, but are not limited to, hydrophilic matrix systems, coated beads or granules, inert plastic matrix systems, complex formation, and diffusion-mediated osmotic pump systems [8]. Despite some progress, oral extendedrelease drug products have not yet reached their full potential in the clinic. Conventional technologies have low drug loading capacity, high production costs, or laborious production steps [4,9]. Consequently, our research aimed to develop oral extended-release capsule formulations using the microsphere drug delivery system. Microspheres are particles often composed of an outer polymeric matrix layer of either synthetic or natural components, such as albumin, gelatin, or poly-lactic-coglycolic acid (PLGA) $[10,11]$. Albumin, the carrier protein used in this present study, is an ideal component of microsphere particles due to its purity, ease of incorporation, stability, modification of drug release, and antigenicity [12]. Microspheres fall in the range of 1-1000 microns in diameter. There are a number of advantages to utilizing microspheres as the technology of choice, including prolonged in vivo half-life, decreased generalized drug toxicity, improved patient compliance, and increased stability [13-18].

*Corresponding author: Duc P Do, Department of Pharmaceutical Sciences, College of Pharmacy, Chicago State University, 9501 South King Drive, Douglas Hall 206, Chicago, IL 60628, USA, Tel: 773-821-2597; Fax: 773-821-2595, E-mail: ddo@csu.edu

Received May 22, 2014; Accepted June 14, 2014; Published June 18, 2014

Citation: Enriquez GG, Orawiec BA, Rizvi SAA, Do DP (2014) Formulation Development and In vitro Evaluation of Oral Extended-release Capsules Containing Biodegradable Microspheres. J Nanomed Nanotechnol 5: 208. doi: 10.4172/2157 7439.1000208

Copyright: (c) 2014 Enriquez GG, et al. This is an open-access article distributed under the terms of the Creative Commons Attribution License, which permits unrestricted use, distribution, and reproduction in any medium, provided the original author and source are credited. 
The main objective of this research was to formulate a polymeric microsphere drug delivery system that will effectively provide extended-release characteristics via an orally administered capsule. Acetaminophen (BCS class III) and ibuprofen (BCS class II) were used as model drugs. A Class II drug has low solubility and high permeability, and a Class III drug has high solubility and low permeability [7]. Microspheres in our study were developed using a quick, one-step spray drying process. Presently, there are a number of methods for the preparation of microspheres, which include solvent evaporation, solvent deposition, phase separation, emulsification, desolvation, and spray drying $[19,20]$. Many of these methods require the use of high temperatures in excess of $110^{\circ} \mathrm{C}$ or organic solvents that can potentially lead to drug degradation and loss of drug efficiency [17,21-23]. In our study, microspheres were prepared using spray-drying. Spray drying is a progressive technology that has been in use in the pharmaceutical industry since the 1940s [24,25]. Spray drying operates based on the atomization of a dispersion of polymer containing the drug of interest in a drying chamber. The liquid dispersion is quickly evaporated in the chamber with the heated incoming air. This process transforms the small atomized droplets into microspheres. Microencapsulation by spray drying can protect the active drug against degradation and provide modified-release of the drug [26,27]. Recently, Martins et al. [28] demonstrated that spray-drying may lead to changes in the physical properties and dissolution characteristics of drug substances to improve therapeutic action. Using spray drying as the method for the production of drug-loaded microspheres, data from our previous in vivo animal studies suggested that enhanced efficacy of drug could be achieved by encapsulating it in a biodegradable albumin polymeric matrix $[14,15]$. The current research focused on a novel approach of designing and formulating extended-release capsule products using a microsphere delivery system. This formulation design should aid in preparing oral extended-release products for efficacious treatment and management of various diseases.

\section{Materials and Methods}

\section{Chemicals}

All chemicals used were of reagent grade or higher quality and were used as received without any further purification. Chemicals were obtained from Fisher Scientific, Inc. (Pittsburgh, PA), unless otherwise noted in the manuscript.

\section{Formulation and Preparation of Microspheres}

To develop optimized extended-release oral drug formulations, various concentrations of BSA (1\%,2\% and 5\%) and glutaraldehyde $(0.05 \%, 0.1 \%, 0.5 \%$ and $1 \%)$ were tested. Bovine serum albumin (BSA) was dissolved in purified water and crosslinked with glutaraldehyde at the desired concentration for 24 hours at room temperature. After optimal crosslinking, sodium bisulfite, in a concentration two times that of the glutaraldehyde, was added to the dispersion and placed on the shaker for approximately 5 minutes to neutralize the excess glutaraldehyde. The model drugs in this investigation were acetaminophen (BCS Class III) and ibuprofen (BCS Class II). Upon completion of the crosslinking process, each model drug was incorporated into the crosslinked BSA dispersion to achieve a desirable loading $(0.5 \%, 1 \%$ and $5 \%)$. Various drug formulations were developed using either acetaminophen or ibuprofen.

The liquid dispersion was then spray dried into microspheres using the following parameters: pump feed rate $2 \%$, inlet temperature $120^{\circ} \mathrm{C}$, outlet temperature $44^{\circ} \mathrm{C}$, aspirator $85 \%$, air flow rate $800 \mathrm{NL}$ per hour.
Blank microspheres were obtained using the described procedures without the addition of drug.

\section{Preparation of capsules containing drug-loaded microspheres}

Drug-loaded microspheres were placed into size \#1 hard gelatin capsules. Each capsule was fully packed and examined for weight variation.

\section{Percent recovery of microsphere formulations}

The percent recovery from spray drying was calculated for each microsphere drug formulation. The percent recovery was calculated by the following equation:

$$
\operatorname{Recovery}(\%)=\frac{\text { total weight of microspheres }}{\text { total initial weight of all components in formulation }} \times 100
$$

\section{Drug encapsulation efficiency from microsphere formulations}

For encapsulation efficiency studies, $1 \mathrm{~g}$ of microspheres containing acetaminophen or ibuprofen were placed in a dialysis bag (MWCO 14 $\mathrm{kDa}$ ) with $4.5 \mathrm{~mL}$ of $\mathrm{PBS}, \mathrm{pH} 7.2$ containing $0.1 \%$ sodium dodecyl sulfate (SDS). The dialysis bag with this molecular weight cutoff effectively blocks the passage of BSA, thus allowing the quantification of drug concentrations. The dispersion was vortexed at regular interval for 20 minutes. At predetermined time points within 24 hours, $2 \mathrm{~mL}$ sample was withdrawn and stored at $4^{\circ} \mathrm{C}$ until analysis. An equal volume of fresh PBS-SDS solution was added to maintain volume. The collected samples were centrifuged at $14,000 \times \mathrm{g}$ for 10 minutes, and the supernatants were analyzed for drug content. Acetaminophen or ibuprofen drug concentrations were measured using a UV-Visible spectrophotometer (Unico, SQ4802, Dayton, NJ) at 243 and $264 \mathrm{~nm}$, respectively. A standard curve for each drug was constructed for the quantification of drug concentration from collected samples. The drug encapsulation efficiency, expressed as a percentage, was calculated for each microsphere formulation by the following equation:

Encapsulation Efficiency $(\%)=\frac{\text { amount of drug inside microspheres }}{\text { theoretical weight of drug before encapsulation }} \times 100$

\section{Characterization studies of microspheres and capsules} containing drug-loaded microspheres

Size and surface properties of microspheres: Scanning electron microscopy (SEM) was used to determine the particle size, size distribution, powder uniformity, and surface morphology of microsphere formulations. For each formulation, microsphere powder was coated with gold prior to putting in the SEM (JEOL USA, Inc., JEOL JSM 6610LV, Peabody, MA).

Zeta potential of microspheres: For each microsphere formulation, $2 \mathrm{mg} / \mathrm{mL}$ powder was dispersed in purified water, loaded into an optical well, and the zeta potential was measured using a Zetasizer (Malvern Instruments, Malvern Zetasizer ZEN1600, Westborough, MA).

Thermal stability of microspheres: Microspheres containing acetaminophen or ibuprofen were characterized for their thermal stability. Calorimetric scans were performed in a DSC Q100 calorimeter (TA Instruments, New Castle, DE) at a heating rate of $10^{\circ} \mathrm{C}$ per hour.

Flowability characteristics of microsphere powder: The flowability of the microsphere powder, using the angle of repose and compressibility index, was determined using the procedures outlined in the United States Pharmacopeia (USP) Chapter 1174 [29]. The angle of repose is the maximum possible angle formed between the horizontal base and the surface of a cone-like pile of powder material. For angle 
of repose measurements, the microsphere powder was allowed to flow through a funnel, and the angle of repose $(\theta)$ was calculated by measuring the height $(\mathrm{h})$ and radius $(\mathrm{r})$ of the pile of the microsphere powder [30,31]:

$$
\theta=\tan ^{-1} \frac{h}{r}
$$

The compressibility index (Carr's index), expressed as a percentage, was calculated based on the bulk density $\left(\rho_{\mathrm{b}}\right)$ and tapped density $\left(\rho_{\mathrm{t}}\right)$ of the microsphere powder [30,32]:

$$
\text { Compressibility } \operatorname{Index}(\%)=\frac{(\text { Tapped Density }- \text { Bulk Density })}{\text { Tapped Density }} \times 100
$$

Uniformity of capsules containing drug-loaded microspheres: The weight variation and content uniformity of capsules containing drug-loaded microspheres were evaluated per USP Chapter 905 guidelines [29]. Weight variation was conducted by weighing 10 empty capsules individually. Each capsule was then filled with the microsphere powder and re-weighed to obtain gross weight. The net weight of the microsphere powder was obtained by subtracting the empty capsule shell weight from the gross weight. The drug content of each capsule was determined from the net weight. For content uniformity tests, the drug content in each of the 10 capsules was determined individually. Drug concentrations were measured using a UV-visible spectrophotometer at $243 \mathrm{~nm}$ and $264 \mathrm{~nm}$ for acetaminophen and ibuprofen, respectively.

Disintegration characteristics of capsules containing drugloaded microspheres: The disintegration time of capsules containing drug-loaded microspheres was conducted in a disintegration tester (Caleva, DIST 20 series, Bedford, NH) using the procedures established by the USP Chapter 701 [29]. Using the basket-rack assembly, one capsule was placed in each of the six tubes of the basket, and the discs were placed on top of capsules. The experiments were conducted at $37^{\circ} \mathrm{C}$ using purified water as the medium. The time taken for the capsules to completely disintegrate was recorded.

Drug release characteristics of capsules containing drug-loaded microspheres: Drug release studies were conducted using a modified USP type 1 dissolution apparatus (Distek dissolution apparatus water bath, Model $2500 \mathrm{C}$, North Brunswick, NJ) at $37^{\circ} \mathrm{C}$ and $75 \mathrm{rpm}$ in 90 $\mathrm{mL}$ of phosphate buffered saline (PBS), $\mathrm{pH}$ 7.2. At predetermined time intervals, $1 \mathrm{~mL}$ samples were taken and an equivalent volume of fresh PBS was added to maintain constant volume.

Drug concentrations from collected samples were measured using a UV-visible spectrophotometer at $243 \mathrm{~nm}$ and $264 \mathrm{~nm}$ for acetaminophen and ibuprofen, respectively. Standard curves were constructed for the quantification of drug concentrations. The Higuchi analysis was carried out by plotting the square root time against percent drug release.

\begin{tabular}{|c|c|c|}
\hline Type of Study & Storage Condition & $\begin{array}{c}\text { Testing Frequency } \\
\text { (months) }\end{array}$ \\
\hline Accelerated & $40^{\circ} \mathrm{C} \pm 2^{\circ} \mathrm{C} / 75 \% \mathrm{RH} \pm 5 \% \mathrm{RH}$ & $0,1,2,3,4,5,6$ \\
\hline Intermediate & $30^{\circ} \mathrm{C} \pm 2^{\circ} \mathrm{C} / 65 \% \mathrm{RH} \pm 5 \% \mathrm{RH}$ & $0,1,2,3,4,5,6$ \\
\hline
\end{tabular}

Table 1: Stability testing conditions of capsules containing drug-loaded microspheres. $\mathrm{RH}=$ relative humidity.

\begin{tabular}{|c|c|c|c|c|}
\hline $\begin{array}{c}\text { Microsphere } \\
\text { Formulations }\end{array}$ & $\begin{array}{c}\text { Average Particle } \\
\text { Size }(\boldsymbol{\mu m})\end{array}$ & $\begin{array}{c}\text { Zeta Potential } \\
(\mathbf{m V} \mathbf{)}\end{array}$ & $\begin{array}{c}\text { Recovery } \\
\mathbf{( \% )}\end{array}$ & $\begin{array}{c}\text { Encapsulation } \\
\mathbf{( \% )}\end{array}$ \\
\hline Blank & $1.5 \pm 0.2$ & $-30.1 \pm 1.2$ & 85 & -- \\
\hline Acetaminophen & $2.0 \pm 0.2$ & $-32.5 \pm 1.5$ & 77 & 85 \\
\hline Ibuprofen & $2.2 \pm 0.3$ & $-30.0 \pm 1.7$ & 70 & 80 \\
\hline
\end{tabular}

Table 2: Characterization of microsphere formulations.

\section{Stability testing of capsules containing drug-loaded microspheres}

Stability testing of capsules containing drug-loaded microspheres was conducted for 6 months under intermediate and accelerated conditions, as depicted in Table 1, per FDA guidelines [33]. The physical appearance and drug content from hard gelatin capsules were evaluated at predetermined test intervals. Drug concentrations were determined as described in the previous sections.

\section{Cell uptake of microspheres}

The uptake of microspheres was determined in human colorectal adenocarcinoma Caco-2 cells (ATCC, Manassas, VA), using previously established procedures $[14,15,34,35]$. Caco-2 cells were grown in DMEM medium, supplemented with $10 \%$ FBS and $1 \%$ penicillin-streptomycin at $37^{\circ} \mathrm{C}$ in a humidified environment of $95 \%$ air and $5 \% \mathrm{CO}_{2}$. The medium was changed every other day. Cells were subcultured after $80 \%$ confluency. Caco- 2 cells were plated into 6 -well plates at 500,000 cells per well and incubated until confluency. The culture medium was replaced with fresh medium. Cells were treated with fluorescaminelabeled microspheres at $1 \mathrm{mg} / \mathrm{mL}$ and $2 \mathrm{mg} / \mathrm{mL}$ drug concentrations. The labeling of microspheres with fluorescamine was performed according to previously established procedures [35]. At designated time points, cells were washed three times with PBS to remove free microspheres and then lysed with $500 \mu \mathrm{L}$ of lysis buffer containing 10 $\mathrm{mM}$ Tris- $\mathrm{HCl}, \mathrm{pH} 8.0,150 \mathrm{mM} \mathrm{NaCl}$, and 1\% Triton X-100. The cell lysate was analyzed for fluorescent intensity using a microplate reader (SpectraFluor Plus, Tecan, San Jose, CA) with excitation at $390 \mathrm{~nm}$ and emission at $465 \mathrm{~nm}$. The uptake of microspheres was expressed as the percentage of fluorescein from cells versus the fluorescein from cultured medium.

\section{Statistical analysis}

For each study, the experiments were repeated twice. Data represent mean $\pm S D(n=3)$.

\section{Results and Discussion}

\section{Size and surface characterization of microspheres}

In the present study, bovine serum albumin (BSA) was used as the polymeric carrier for the microsphere drug delivery system. BSA is used as a carrier due to its purity, ease of drug incorporation, desired drug release, drug stability and antigenicity [12]. Polymeric microsphere formulations were prepared by spray-drying and were characterized for various properties. Representative properties of microspheres from spray drying are shown in Table 2 . The percent recovery of microsphere formulations was from $70 \%-85 \%$. This high percentage of recovery is important in the large scale production of microsphere drug formulations. The encapsulation efficiency was calculated to be from $80-85 \%$, suggesting that spray drying is an effective method for preparing drug formulations with high drug loading capacity.

Scanning electron microscopy was used to determine the size, size distribution, uniformity, and surface morphology of the drug-loaded microsphere formulations. Each of these physicochemical factors has a profound effect on the release characteristics of the drug from the delivery system. The SEM analysis indicated that microspheres were spherical and uniform in shape (Figure 1), which is critical for maintaining constant drug release properties. The average size of microspheres was approximately $2 \mu \mathrm{m}$ (microns) in size. Champion et al. [36] showed that particles with a diameter of 2-3 $\mu \mathrm{m}$ exhibited optimal 


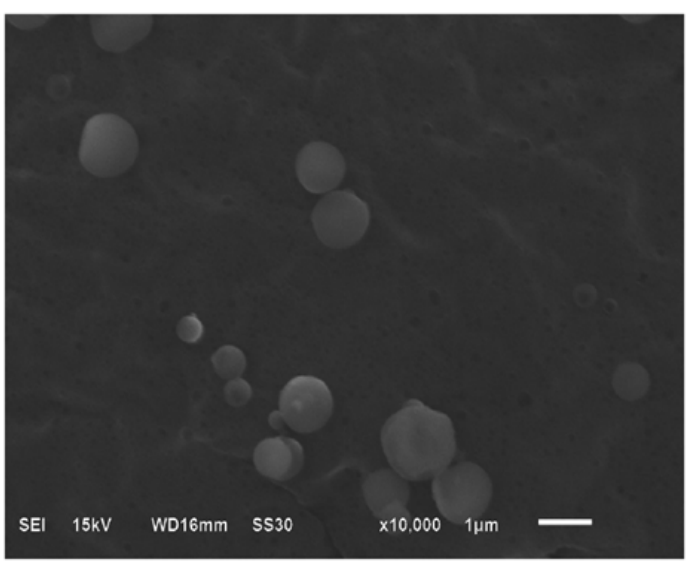

Figure 1: Representative microscopic image of blank microspheres at $10,000 x$ magnification. The microsphere powder was coated with gold prior to SEM analysis.

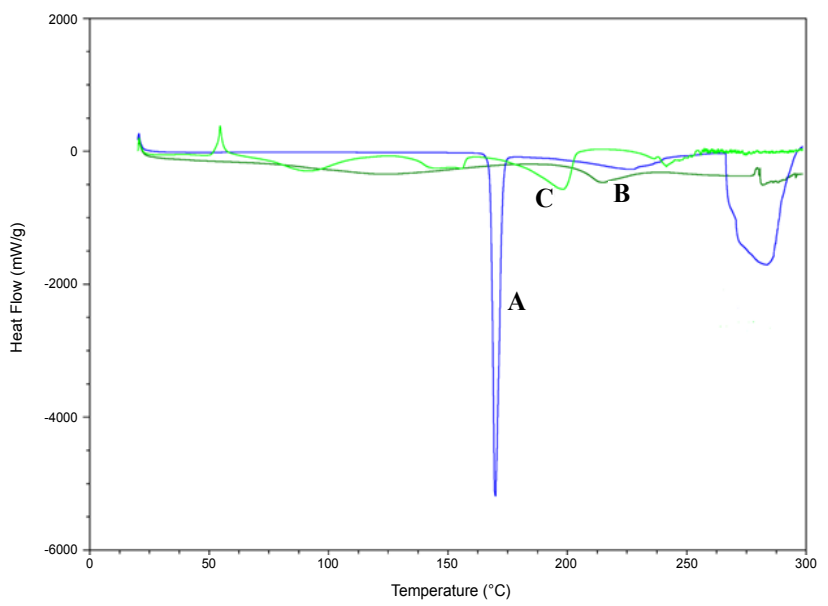

Figure 2a: Differential scanning calorimetry thermogram of acetaminophenloaded microspheres. (A): acetaminophen; (B): bovine serum albumin; and (C): acetaminophen-loaded microspheres.

phagocytosis. Additionally, our data showed that the Zeta potential was $-32.5 \mathrm{mV}$ and $-30.0 \mathrm{mV}$ for acetaminophen and ibuprofen-loaded microspheres, respectively. Zeta potential measurements provide surface properties and stability information of the microspheres. These values indicated that formulated microspheres are stable in aqueous environments. Particles with Zeta potential of $\pm 30 \mathrm{mV}$ are considered stable [37-39].

\section{Thermal stability characterization of drug-loaded microspheres}

A critical component in the development of drug-loaded microspheres is ensuring that the ingredients are not degraded when subjected to spray drying. Thermal properties of drug formulations indicate their overall stability; thus changes in the stability of a drug during the formulation and preparation processes can be detected by examining the changes in the thermal properties of the formulations. In our study, thermal stability was assessed using differential scanning calorimetry (DSC). Calorimetric scans were performed at a heating rate of $10^{\circ} \mathrm{C}$ per hour. Representative thermograms of acetaminophen and ibuprofen are shown in Figure 2A and 2B, respectively. In the heat flow thermogram, thermal transitions appear as peaks, and the higher the $\mathrm{T}_{\mathrm{m}}$ (temperature at which $50 \%$ of the substance is degraded), the more stable the formulation. Our data indicated that the drug substance remains stable throughout the microencapsulation process by spray drying, as indicated by an increase in the melting point temperature $\left(\mathrm{T}_{\mathrm{m}}\right)$.

\section{Flowability characteristics of drug-loaded microspheres}

The objective of our research was to develop oral capsule formulations using microspheres as the platform technology for providing extended-release. As a result, drug-loaded microsphere powder was characterized for its flowability. These parameters are crucial in the large scale manufacturing of capsule dosage forms. For flowability, the angle of repose and compressibility index were measured. Formulated microspheres showed an average angle of repose of $28^{\circ} \pm$ $1^{\circ}$. A powder possessing an angle of repose of $25^{\circ}$ to $30^{\circ}$ is considered to have "excellent" flow property [29]. Compressibility of the drug-loaded microspheres was determined by the Carr's (compressibility) index. Drug-loaded microspheres had an average compressibility index of $21 \% \pm 0.5 \%$, thus indicating a "passable" flowability characteristics [29].

\section{Uniformity and disintegration rate of capsules containing drug-loaded microspheres}

Uniformity of capsules containing drug-loaded microspheres was demonstrated by the weight variation and the content uniformity tests. Capsules were uniform with regards to weight $(99.5 \% \pm 0.5 \%)$ and drug content $(99.5 \% \pm 0.2 \%)$; thus, they met the uniformity tests based on the USP guidelines [29]. Additionally, the average disintegration time for capsules was 20 minutes \pm 3 minutes, which are consistent with the disintegration rate of oral dosage forms.

\section{Release studies of microspheres and capsules containing drug-loaded microspheres}

In our study, acetaminophen and ibuprofen were used as model drugs. As shown in Figures $3 \mathrm{~A}$ and $3 \mathrm{~B}$, the microsphere powder displayed a steady release of the drug for 40 hours. Figures $3 \mathrm{~A}$ and $3 \mathrm{~B}$ show the comparative release profiles of acetaminophen- and ibuprofenloaded microsphere formulations, respectively. For both ibuprofen and

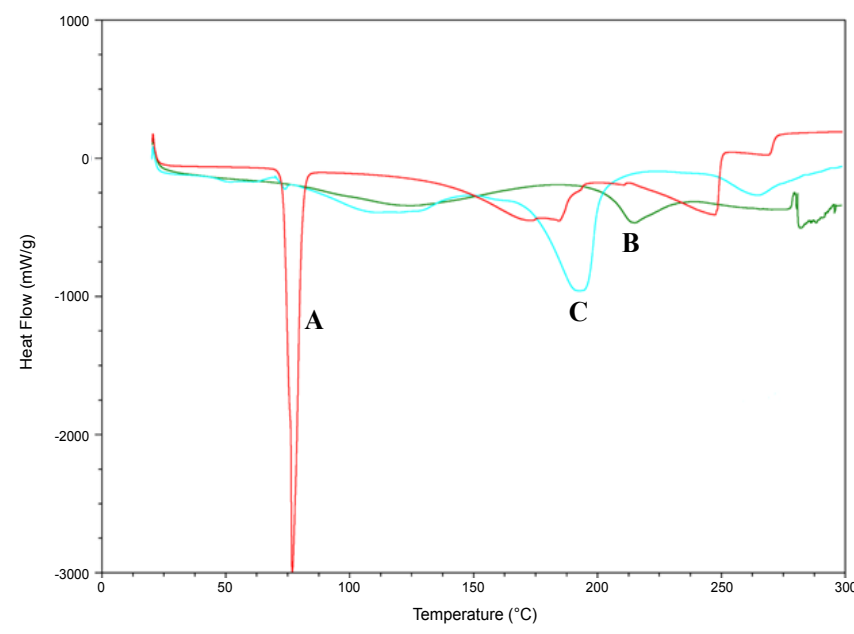

Figure 2b: Differential scanning calorimetry thermogram of ibuprofenloaded microspheres. (A): ibuprofen; (B): bovine serum albumin; and (C): ibuprofen-loaded microspheres. 


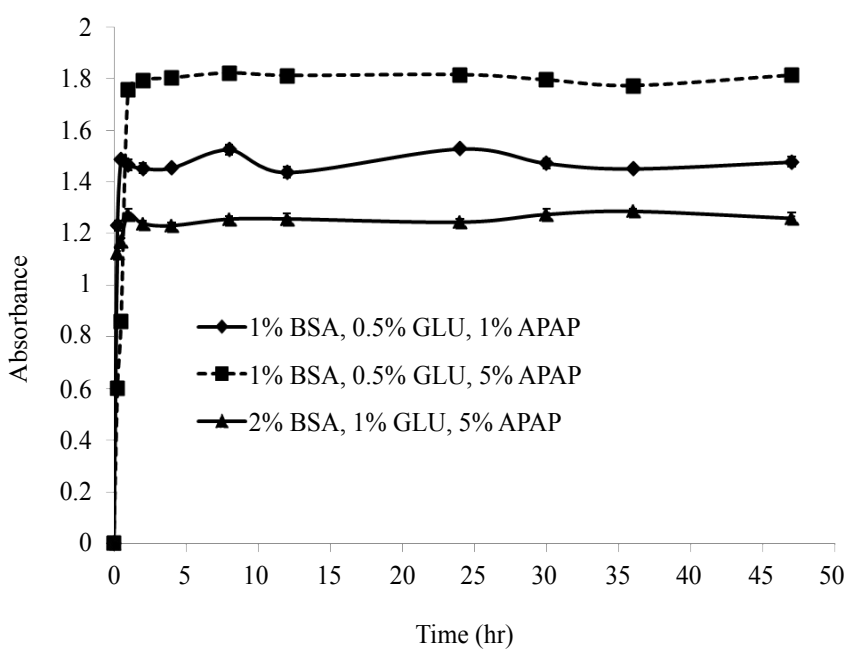

Figure 3a: Release profiles of microsphere formulations containing various concentrations of acetaminophen. $\mathrm{GLU}=$ glutaraldehyde; $\mathrm{BSA}=$ bovine serum albumin; APAP = acetaminophen. Data represent mean $\pm \operatorname{SD}(n=3)$.

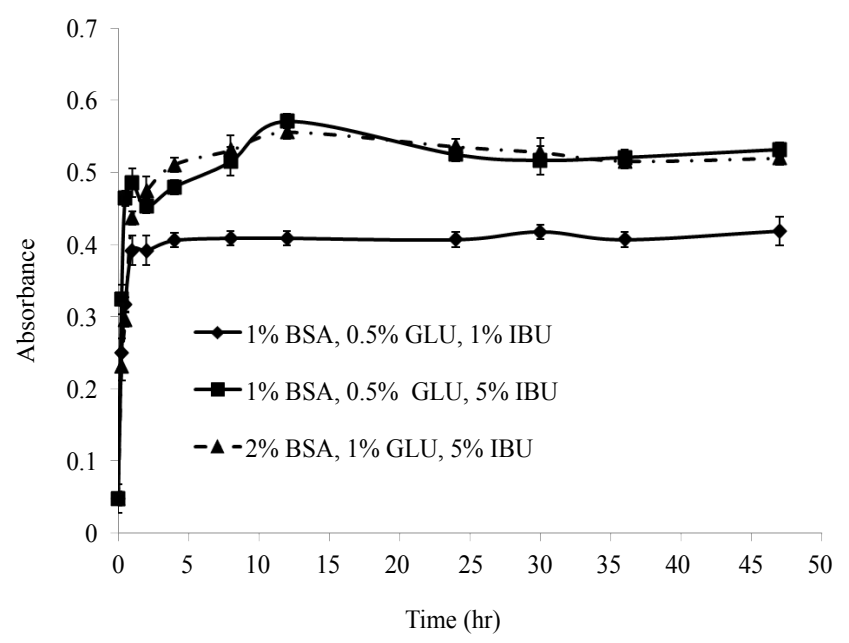

Figure 3b: Release profiles of microsphere formulations containing various concentrations of ibuprofen. GLU=glutaraldehyde; BSA=bovine serum albumin; IBU = ibuprofen. Data represent mean \pm SD $(n=3)$

acetaminophen formulations, our data indicated that microspheres could be loaded with either a $1 \%$ or $5 \%$ drug concentration and still maintain an extended-release profile. All microsphere formulations provided an initial burst release, which is highly desired to provide an immediate dose of the drug in systemic circulation. The initial burst release was followed by maintenance of extended release of the drug substance for about 40 hours.

Prepared drug-loaded microsphere formulations were incorporated into hard gelatin capsules as the goal of the present study was to deliver microspheres using a conventional capsule dosage form. Capsules containing drug-loaded microspheres were evaluated for their release characteristics. Figure $4 \mathrm{~A}$ depicts an initial burst release, followed by a steady release of acetaminophen for 16 hours from capsules containing acetaminophen-loaded microspheres. The best formulation was determined to be $1 \% \mathrm{BSA} / 0.05 \%$ glutaraldehyde as it displayed the highest absorbance of the drug. Similarly, 1\% BSA / 0.05\% glutaraldehyde displayed the highest absorbance for capsules containing ibuprofenloaded microspheres (Figure 4B). Glutaraldehyde could be used at $0.05 \%, 0.1 \%$ or $0.5 \%$ when the polymeric BSA concentration is $1 \%$. If a higher BSA concentration is required, the glutaraldehyde concentration should be optimized to provide the most desirable release profile.

Overall, our release data showed an initial burst release followed by a steady release of drug for approximately 40 hours from microspheres, indicating that the delivery system was effective in the release of drug over time. The release characteristics of the capsule formulations

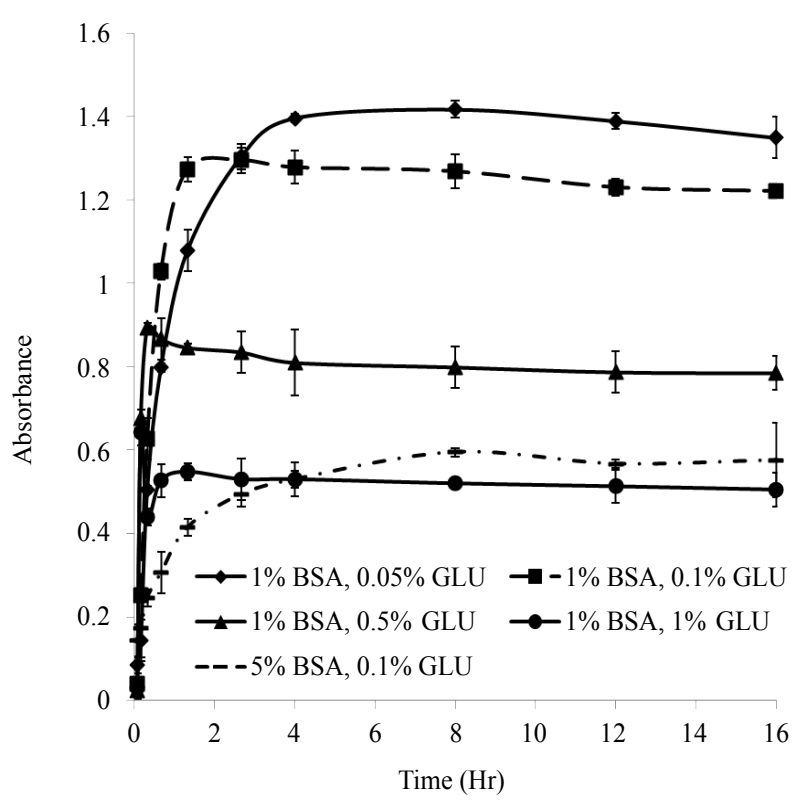

Figure 4a: Release studies of capsules containing acetaminophen-loaded microspheres over a period of 16 hours. Each formulation contained $0.5 \%$ acetaminophen. BSA=bovine serum albumin; $G L U=$ glutaraldehyde. Data represent mean $\pm S D(n=3)$.

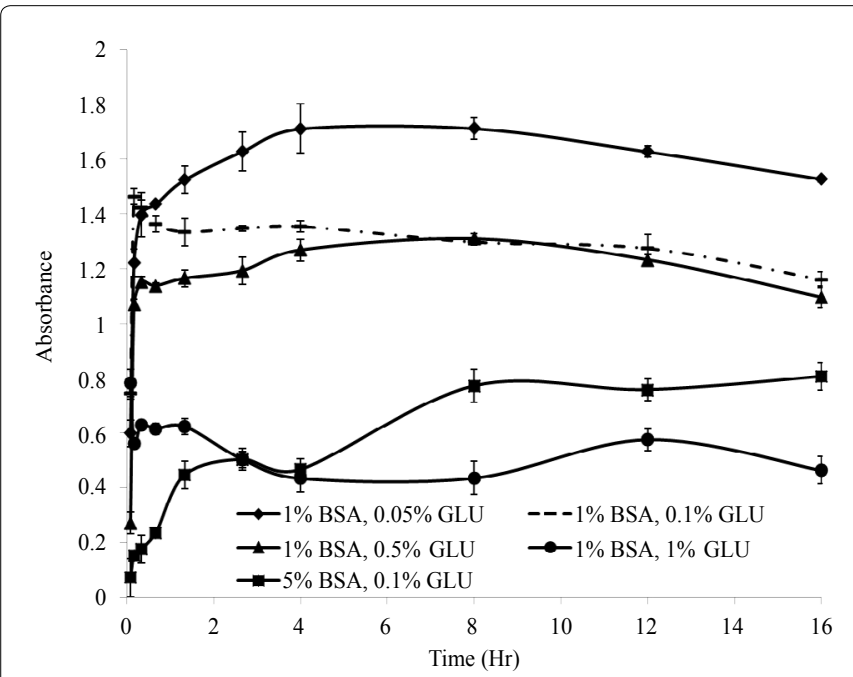

Figure 4b: Release studies of capsules containing ibuprofen-loaded microspheres over a period of 16 hours. Each formulation contained $0.5 \%$ ibuprofen. BSA = bovine serum albumin; GLU = glutaraldehyde. Data represent mean $\pm \operatorname{SD}(n=3)$. 


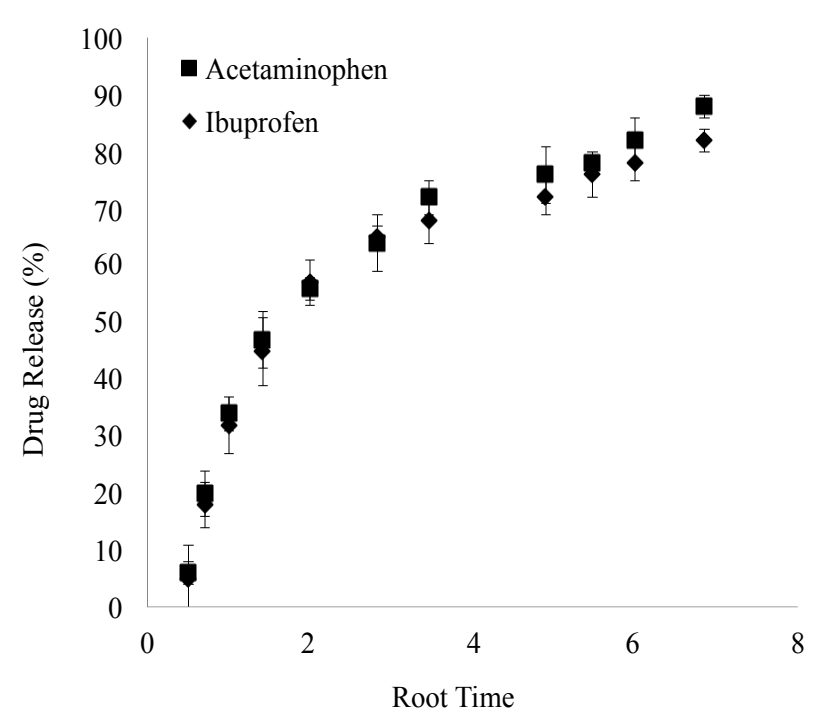

Figure 5: Higuchi analysis of drug from capsules containing drug-loaded microspheres. Higuchi analysis was carried out by plotting the square root time against percent drug release. Data represent mean $\pm S D(n=3)$

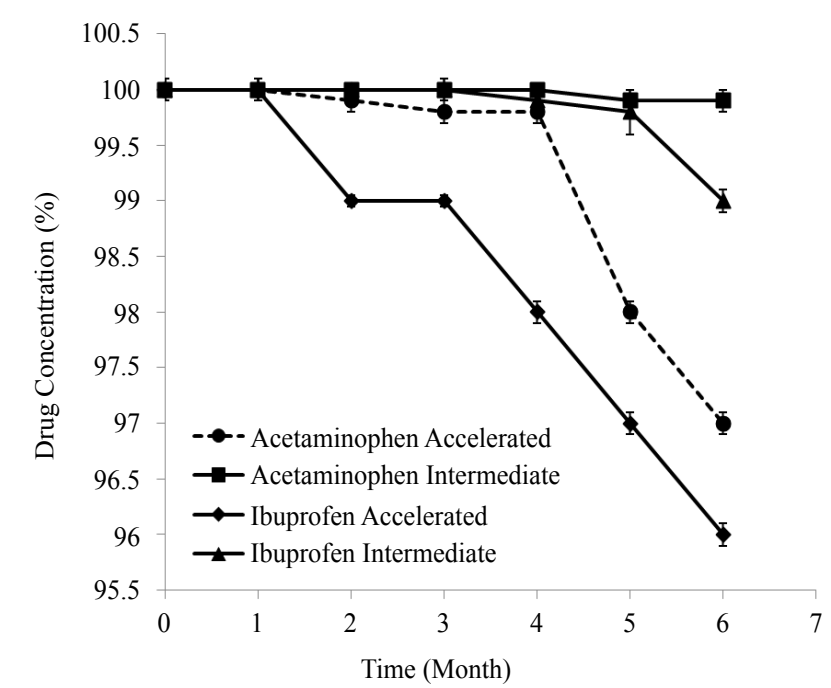

Figure 6: Stability testing of capsules containing acetaminophen- and ibuprofen-loaded microspheres under intermediate and accelerated conditions. Data represent mean $\pm S D(n=3)$.

containing drug-loaded microspheres were further investigated and depicted using the Higuchi model. Figure 5 shows an extended release profile of drug after an initial burst release.

\section{Stability testing of capsules containing drug-loaded microspheres}

Stability testing of capsules containing drug-loaded microspheres was conducted for 6 months at intermediate and accelerated conditions to establish a suitable formulation for drug delivery. Stability testing provides information regarding the long-term stability as well as the degradation behavior of the drug and formulations. Our data indicated that after 6 months, both acetaminophen and ibuprofen formulations maintained at least $96 \%$ of their original concentrations (Figure 6).

\section{Uptake of biodegradable microspheres in Caco-2 cells}

The uptake of biodegradable microspheres was conducted in intestinal Caco- 2 cells at $1 \mathrm{mg} / \mathrm{mL}$ and $2 \mathrm{mg} / \mathrm{mL}$ concentrations. The percentage of uptake efficiency in cells was similar between the two concentrations used (Figure 7). Additionally, at either concentration, there was a time-dependent uptake of microspheres into cells with approximately $25 \%$ uptake efficiency at 24 hours. These data suggested that formulated microspheres were able to be internalized into cells.

\section{Conclusion}

Previously, we showed that microspheres can be formulated to provide controlled-release of parenteral anticancer drug preparations in the in vivo environment, thus leading to enhanced efficacy $[14,15]$. The objective of the current study was to develop extended-release capsules containing biodegradable microspheres to attain effective therapeutic concentration of drug. Ibuprofen (BCS class II) and acetaminophen (BCS class III) were used as model drugs to assess the suitability and versatility of the spray dried microsphere system for extended-drug delivery. A Class II drug has low solubility and high permeability, and a Class III drug has high solubility and low permeability, thus the development of extended-release formulations for these classes of drugs is desirable.

Drug-loaded microspheres were prepared by a microencapsulation method via spray-drying and were incorporated into hard gelatin capsules. Our data suggested that drug-loaded microspheres can be prepared using a quick one-step spray drying process without the use of organic solvents. Additionally, release studies of capsule formulations containing drug-loaded microspheres resulted in extended release of the model drugs for 16 hours in vitro. Differential scanning calorimetry data indicated that the integrity of the drug remained throughout the microencapsulation process. The formulated microspheres were shown to be capable of internalization by intestinal cells. These data provided evidence for the role of the microsphere delivery system in the extended-release delivery of BCS Class II and III drug substances. The microsphere delivery system shows potential for the development of oral, extended-release pharmaceutical dosage forms aiming at

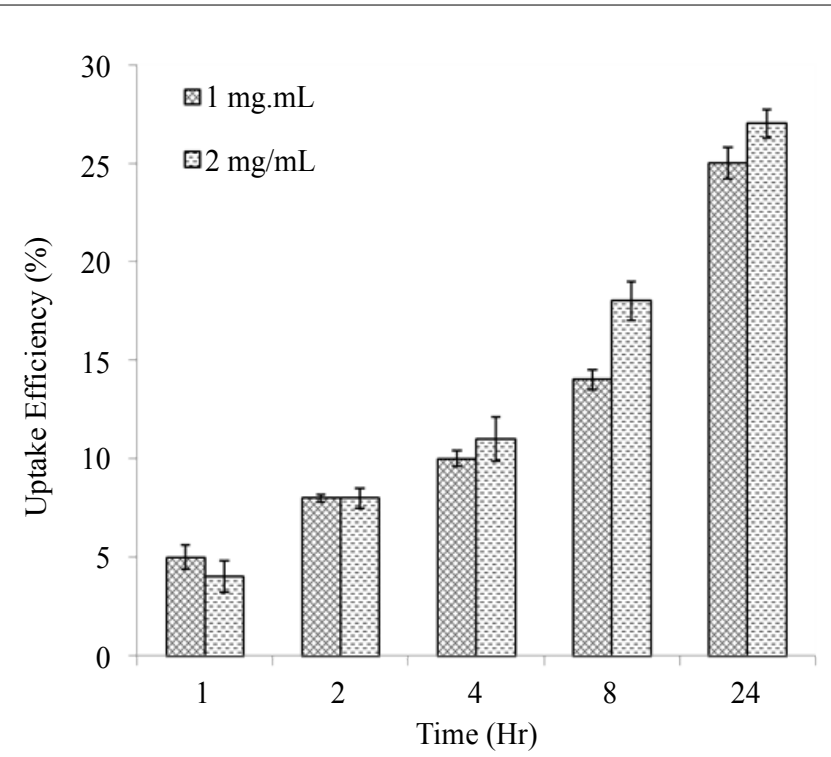

Figure 7: The uptake efficiency of microspheres in Caco-2 cells at $1 \mathrm{mg} / \mathrm{mL}$ and $2 \mathrm{mg} / \mathrm{mL}$ concentrations. Data represent mean $\pm \mathrm{SD}(\mathrm{n}=3)$. 
Citation: Enriquez GG, Orawiec BA, Rizvi SAA, Do DP (2014) Formulation Development and In vitro Evaluation of Oral Extended-release Capsules Containing Biodegradable Microspheres. J Nanomed Nanotechnol 5: 208. doi: 10.4172/2157-7439.1000208

improved bioavailability of conventional drugs and minimizing side effects.

\section{Acknowledgement}

The authors would like to acknowledge the invaluable assistance of Lakshmi Prasanna Kolluru and Martin J. D'Souza (Mercer University, Atlanta, GA, USA) in the data collection of DSC samples.

\section{References}

1. Bernkop-Schnürch $A$ (2013) Nanocarrier systems for oral drug delivery: do we really need them? Eur J Pharm Sci 49: 272-277.

2. Jain A, Jain A, Gulbake A, Shilpi S, Hurkat P, et al. (2013) Peptide and protein delivery using new drug delivery systems. Crit Rev Ther Drug Carrier Syst 30: 293-329.

3. Yamashita F, Hashida M (2013) Pharmacokinetic considerations for targeted drug delivery. Adv Drug Deliv Rev 65: 139-147.

4. Birkett DJ (1998) Pharmacokinetics made easy. McGraw Hill, Sydney, Australia.

5. Diab R, Jaafar-Maalej C, Fessi H, Maincent P (2012) Engineered nanoparticulate drug delivery systems: the next frontier for oral administration? AAPS J 14: 688-702.

6. Kaur T, Gill B, Kumar S, Gupta G (2011) Mouth dissolving tablets: a nove approach to drug delivery. Int J Curr Pharm Res 3: 1-7.

7. Pang J, Luan Y, Li F, Cai X, Du J, et al. (2011) Ibuprofen-loaded poly(lactic-coglycolic acid) films for controlled drug release. Int J Nanomedicine 6: 659-665.

8. Allen L, Popovich N, Ansel H (2011) Pharmaceutical dosage forms and drug delivery systems. (8thedn), Lippincott Williams \& Wilkins, Baltimore, MD.

9. Das N, Das S (2003) Controlled-release of oral dosage forms. Formulation, Fill \& Finish 10-16

10. Hari BN, Praneetha T, Prathyusha T, Mounika K, Devi DR (2012) Development of starch-gelatin complex microspheres as sustained release delivery system. J Adv Pharm Technol Res 3: 182-187.

11. Okada H, Toguchi H (1995) Biodegradable microspheres in drug delivery. Crit Rev Ther Drug Carrier Syst 12: 1-99.

12. Thakkar H, Sharma RK, Mishra AK, Chuttani K, Murthy RR (2005) Albumin microspheres as carriers for the antiarthritic drug celecoxib. AAPS PharmSciTech 6: E65-73.

13. Alcalá-Alcalá S, Urbán-Morlán Z, Aguilar-Rosas I, Quintanar-Guerrero D (2013) A biodegradable polymeric system for peptide-protein delivery assembled with porous microspheres and nanoparticles, using an adsorption/infiltration process. Int J Nanomedicine 8: 2141-2151.

14. Do DP, Pai SB, Rizvi SA, D'Souza MJ (2010) Development of sulforaphaneencapsulated microspheres for cancer epigenetic therapy. Int J Pharm 386: 114-121.

15. Enriquez GG, Rizvi SA, D'Souza MJ, Do DP (2013) Formulation and evaluation of drug-loaded targeted magnetic microspheres for cancer therapy. Int J Nanomedicine 8: 1393-1402.

16. Jyothi S, Seethadevi A, Suria P, Muthuprasanna P, Pavitra P (2012) Microencapsulation: a review. Int J of Pharma \& Bio Sciences 3: 509-531.

17. Kettenbach J, Stadler A, Katzler IV, Schernthaner R, Blum M, et al. (2008) Drug-loaded microspheres for the treatment of liver cancer: review of current results. Cardiovasc Intervent Radiol 31: 468-476.

18. Rajput MS, Agrawal P (2010) Microspheres in cancer therapy. Indian J Cancer 47: 458-468.

19. Das S, Chaudhury A (2011) Recent advances in lipid nanoparticle formulations with solid matrix for oral drug delivery. AAPS PharmSciTech 12: 62-76.
20. Malik DK, Baboota S, Ahuja A, Hasan S, Ali J (2007) Recent advances in protein and peptide drug delivery systems. Curr Drug Deliv 4: 141-151.

21. Ikeda M (1992) Public health problems of organic solvents. Toxicol Lett 64-65 Spec No: 191-201.

22. Jang J, Sah H (2011) Nonhalogenated solvent-based solvent evaporation process useful in preparation of PLGA microspheres. J Microencapsul 28: 490 498

23. Spencer PS, Schaumburg HH (1985) Organic solvent neurotoxicity. Facts and research needs. Scand J Work Environ Health 11 Suppl 1: 53-60.

24. Johansen P, Merkle HP, Gander B (2000) Technological considerations related to the up-scaling of protein microencapsulation by spray-drying. Eur J Pharm Biopharm 50: 413-417.

25. Snyder HE (2012) Pharmaceutical spray drying: solid-dose process technology platform for the 21st century. Ther Deliv 3: 901-912.

26. Pavanetto F, Genta I, Giunchedi P, Conti B, Conte U (1994) Spray-dried albumin microspheres for the intra-articular delivery of dexamethasone. $J$ Microencapsul 11: 445-454.

27. Schrooyen PM, van der Meer R, De Kruif CG (2001) Microencapsulation: its application in nutrition. Proc Nutr Soc 60: 475-479.

28. Martins R, Machado M, Pereira S, Nosari A, Tacon L, et al. (2012) Engineering active pharmaceutical ingredients by spray drying: effects on physical properties and in vitro dissolution. Dry Technol 30: 905-913.

29. United States Pharmacopeia and the National Formulary (USP 35-NF 30) (2012) The United States Pharmacopeial Convention, Rockville, MD.

30. Aslani A, Jahangiri $H$ (2013) Formulation, characterization and physicochemica evaluation of ranitidine effervescent tablets. Adv Pharm Bull 3: 315-322.

31. Gunn C, Carter S (1986) Cooper and Gunn's tutorial pharmacy. New Delhi, CBS Publishers, New Delhi, India.

32. Nagar P, Singh K, Chauhan I, Verma M, Yasir M (2011) Orally disintegrating tablets: Formulation, preparation techniques and evaluation. J Appl Pharm Sci 1: $35-45$.

33. Guidance for industry: Q1a(r2) stability testing of new drug substances and products. US Food and Drug Administration.

34. Luo Y, Teng Z, Wang TT, Wang Q (2013) Cellular uptake and transport of zein nanoparticles: effects of sodium caseinate. J Agric Food Chem 61: 7621-7629.

35. Nettey H, Haswani D, D'Souza M, Oettinger C (2007) In vitro antimicrobial effect of encapsulated vancomycin on internalized Staphylococcus aureus within endothelial cells. Drug Dev Ind Pharm 33: 133-139.

36. Champion JA, Walker A, Mitragotri S (2008) Role of particle size in phagocytosis of polymeric microspheres. Pharm Res 25: 1815-1821.

37. Everett D (1994) Basic principles of colloid science. The Royal Society of Chemistry, UK

38. Lyklema J (1991) Fundamentals of interface and colloid science. Academic Press, UK.

39. Shaw D (1992) Introduction to colloid and surface chemistry. Butterworth Heinemann, UK. 